\title{
Study of Umbilical Cord in Normal and Intra Uterine Growth Retardation (IUGR) Placenta
}

\author{
Pooja Dhabhai ${ }^{1}$, Ghanshyam Gupta르, Harish Kumar Gurjer ${ }^{3}$ \\ ${ }^{1}$ Department of Anatomy, RNT Medical College, Udaipur, Rajasthan, India. ${ }^{2}$ Department \\ of Anatomy, RNT Medical College, Udaipur, Rajasthan, India. ${ }^{3}$ Department of Medical \\ and Health, Udaipur, Rajasthan, India.
}

\section{ABSTRACT}

\section{BACKGROUND}

The purpose of the study is to compare the gross anatomy of umbilical cord in normal placentas and IUGR placentas.

\section{METHODS}

This is a case control study, conducted in the Department of Anatomy, R.N.T. Medical College, Udaipur among 100 control and 100 IUGR placentas. Placenta was collected as soon as possible after delivery and was examined for gross changes. Then it was fixed in $10 \%$ formalin for sections for staining.

\section{RESULTS}

In our study, the marginal attachment of umbilical cord with 0.25 insertion percentage was seen in 35 cases. The association between marginal attachment of the cord and low birth weight is statistically significant.

\section{CONCLUSIONS}

Increased incidence of marginal cord insertion was associated with low foetal weight. The mean length of umbilical cord varied in the present study between $30 \mathrm{cms}$ and $60 \mathrm{cms}$ in control and IUGR group. No significant difference was seen in the cord length according to type of pregnancy, normal or abnormal. From the literature review, it was anticipated that there would be a wide range of umbilical cord lengths. The umbilical cord may have a central, lateral, marginal or medial insertion. Central and lateral insertions have no clinical importance. Marginal insertions may be more susceptible to vessel rupture and have been associated with foetal growth retardation, stillbirth and neonatal death.

\section{KEY WORDS}

Infarction, Placenta, Intra-Uterine Growth Retardation
Corresponding Author:

Dr. Pooja Dhabhai,

Flat No. 304, Satyam Estate,

Block-A, Goverdhanvilas, Balicma,

Udaipur, Rajasthan, India.

E-mail: poojadhabhai14@gmail.com

DOI: $10.14260 / \mathrm{jemds} / 2020 / 565$

How to Cite This Article:

Dhabhai P, Gupta G, Gurjer HK, et al. Study of umbilical cord in normal and intrauterine growth retardation (IUGR) placenta. J Evolution Med Dent Sci 2020;9(36):2597-2600, $10.14260 / \mathrm{jemds} / 2020 / 565$

Submission 07-03-2020,

Peer Review 14-07-2020,

Acceptance 20-07-2020,

Published 07-09-2020.

Copyright (c) 2020 JEMDS. This is an open access article distributed under Creative Commons Attribution License [Attribution 4.0 International (CC BY 4.0)] 


\section{BACKGROUND}

Placenta is the most important accessory foetal structure that brings foetal and maternal circulation into close relationship. Morphologically it is partly of foetal origin - the trophoblast, and partly of maternal origin-the decidua (Pansky B). ${ }^{1}$ The examination of placenta (like ultrasonography or chorionic villi biopsy etc.) is one of the common methods of investigation for factors endangering the foetus and the newborn.

Nowadays, scientists are taking greater interest in the placenta and its function than ever before. One reason for this is that with great improvements in mechanistic management in obstetrics and advances in neonatal care, foetal death in Utero, the great majority of which can only be described to 'Placental insufficiency', represents an ever-increasing proportion of perinatal mortality. Placental insufficiency means insufficient exchange or metabolic efficiency of this organ for the growing foetus. The placenta should not be considered as an isolated, autonomically functioning organ but rather as an integral part of a maternofoetal or foetoplacental functional unit. The extension of the concept "placental insufficiency syndrome" is in line with such a concept. Pathophysiologically we can differentiate between three main forms of insufficiency - haemodynamic placental insufficiency, ${ }^{2}$ insufficiency of the placental membrane ${ }^{3}$ and cellular parenchymatous insufficiency. The haemodynamic placental insufficiency must be considered as an overriding principle of the insufficiency of all partial functions of the placenta. It is probable that the intrauterine growth of the foetus depends mainly on the placental blood flow factors, the oxygen uptake appearing to be the actual limiting factor. For clinical purposes it is advisable to follow the well-tried principle of dividing the placental insufficiency syndrome into three parts namely-a chronic, a subchronic and an acute form.

The concept of "placental insufficiency" appears to offer a plausible explanation for foetal emergency situations; in reality however, it leads to unproven and possibly wrong ideas on the genesis of these phenomena. It is applied in a semantically inconsistent manner and that is one of the reasons why it is unsuitable as a term that can be used in scientific language. It is nevertheless being continually used in present-day "medicalese" and even in recent textbooks of obstetrics as a definition of nosological entities. The claim that the term "placental insufficiency" is necessary for mutual communication in obstetrics is not substantiated or, if at all, with the self-confirming claim that it cannot be eliminated because it is already firmly rooted in clinical thinking. The conditions in the foetus and the newborn labelled "placental insufficiency syndrome" could be defined without additional linguistic effort by means of a number of sufficiently precise and far more important terms, namely "underweight", "dystrophy", "asphyxia/hypoxia". These terms stand for results of direct observation without hypothetical connotations. The conditions they describe are at least in principle objectifiable and measurable. The expressions allow and induce further deliberations and studies on actual causes of chronic or acute deficient supply of the foetus, the nascent and newborn infant, and how to avoid these causes. Intrauterine growth retardation (IUGR), often associated with functional placental insufficiency, results in increased perinatal mortality and morbidity. For obvious reasons, many questions regarding the progression of IUGR pregnancies cannot be addressed experimentally in humans, predicating the use of animal models. Although no animal model fully recapitulates human pregnancy (Wulf KH 1981). ${ }^{2}$

Human placenta is haemochorial, that is, maternal blood is in direct contact with the chorionic epithelium. During its relatively short life span it serves a wide range of functions.

There can be no doubt that as an organ it is unique. No other organ in the body contains both maternal and foetal tissues. Hence the study of placenta presents a uniquely efficient opportunity to obtain information about two individuals - the mother and the foetus.

Normal foetal growth is a critical component of a healthy pregnancy and influences the long-term health of the offspring. However, defining normal and abnormal foetal growth has been a long-standing challenge in clinical practice and research.

IUGR is a relatively common problem and a potential cause of significant perinatal morbidity and mortality. There are many possible causes of IUGR, and some cases may involve more than one. Although an underlying cause may not always be identifiable, it is important to consider all the factors because identification of the underlying cause of the IUGR may impact short-term management as well as long-term prognosis. The cornerstone of management for IUGR involves close antenatal surveillance with a well-timed delivery.

\section{METHODS}

This is a case control study carried out at R.N.T. Medical College \& Hospital, Udaipur. Placentae were collected from 200 women admitted and delivered in Mahila Chikitsalaya attached to R.N.T. Medical College, Udaipur. Cases taken were those admitted to the labour rooms of the hospital (either directly or through the antenatal wards). The cases were selectively studied between the period 1-7-2013 to 1-4-2014. All the cases were within the age group of 18-40 years, of average height and weight and included both primigravida and multigravida. All the cases were free from any other systemic disease. Simple random sampling was done. Sample size was decided according to the number of samples (normal and IUGR) we were able to get in that given period of time 1-72013 to 1-4-2014.

\section{Group 1 - Normal Pregnancy}

100 patients included in this group had a haemoglobin above $9 \mathrm{~g} / \mathrm{dl}$ and a normal urine analysis-not associated with any disease.

\section{Group 2 - IUGR Cases}

100 cases of Idiopathic IUGR were included.

\section{Methods}

After the delivery placentae were collected for morbid and histopathological studies. The size, shape, surface area, weight of placenta was noted along with the inspection of marginal veins for any thrombus. The number of cotyledons, condition of membranes, and presence of infarction, calcification and 
length and site of insertion of umbilical cord were noted. Gross examination of placentae was done as soon as possible after the delivery. Membranes were then trimmed from the placental margin. Umbilical cord was cut $4 \mathrm{cms}$ from the site of insertion. Placenta was washed and surface dried between blotting papers. Weight of placenta was taken after removing the membranes and cutting the cord leaving a $4 \mathrm{cms}$ stub above its placental insertion and removing the blood clots. Surface area of the placenta was recorded by cutting out its shape on a piece of plastic sheet which was mapped on a graph sheet to calculate the area. Assuming the placenta to be a perfect circle the mean radius ' $r$ ' was estimated from the surface area. The minimum distance between the site of insertion of umbilical cord and the margin of the placenta was measured and denoted as ' $d$ '. The insertion percentage $d / r$ x 100 was then worked out. Low insertion percentage implies a marginal insertion, while a high insertion percentage indicates a centrally attached umbilical cord. Weight of the placenta was taken and its surface area, foetal surface, maternal surface, amnion membranes were examined. Umbilical cord was examined for its length and insertion.

\section{Inclusion Criteria}

100 placentas from normal control pregnancies and 100 placentas from mother who delivered IUGR babies.

\section{Exclusion Criteria}

We referred to only uncomplicated pregnancies without any previous diseases.

\section{Statistical Analysis}

Results were expressed in frequency and percentage. Significance was assessed at 5\% level of significance. Chisquare was used to find the significance of study parameters on categorical scale. P-value of 0.05 or less was considered for statistical significance. SPSS version 10 was used for the analysis of the data and Microsoft Word and Excel were used to generate graphs and tables.

\section{RESULTS}

\section{Normal Placenta}

\begin{tabular}{|ccc|}
\hline Length of Cord & No. of Placenta & \% of Placenta \\
$30-40 \mathrm{~cm}$ & 35 & 35 \\
$41-50 \mathrm{~cm}$ & 51 & 51 \\
$51-60 \mathrm{~cm}$ & 14 & 14 \\
\hline \multicolumn{3}{|c|}{ Table 1.1. Umbilical Cord Length } \\
\hline
\end{tabular}

\begin{tabular}{|ccc|}
\hline Insertion & No. of Placenta & \% of Placenta \\
Central & 38 & 38 \\
Lateral & 25 & 25 \\
Marginal & 18 & 18 \\
Medial & 19 & 19 \\
\hline \multicolumn{3}{|c}{ Table 1.2. Insertion of Umbilical Cord } \\
\hline
\end{tabular}

In our study, the marginal attachment of umbilical cord with 0.25 insertion percentage was seen in 35 cases. The association between marginal attachment of the cord and low birth weight is statistically significant. The mean length of umbilical cord varied in the present study.

\section{IUGR Placenta}

\begin{tabular}{|ccc|}
\hline Length of Cord & No. of Placenta & \% of Placenta \\
$30-40 \mathrm{~cm}$ & 33 & 33 \\
$41-50 \mathrm{~cm}$ & 33 & 33 \\
$51-60 \mathrm{~cm}$ & 34 & 34 \\
\hline \multicolumn{3}{|c|}{ Table 2.1. Umbilical Cord Length } \\
\hline
\end{tabular}

\begin{tabular}{|ccc|}
\hline Insertion & No. of Placenta & \% of Placenta \\
Central & 29 & 29 \\
Lateral & 22 & 22 \\
Marginal & 35 & 35 \\
Medial & 14 & 14 \\
\hline \multicolumn{3}{|c|}{ Table 2.2. Insertion of Umbilical Cord } \\
\hline
\end{tabular}

\begin{tabular}{|cccccc|}
\hline Variable & \multicolumn{2}{c}{$\begin{array}{c}\text { Normal Pregnancies } \\
\text { Group (n= 100) }\end{array}$} & \multicolumn{2}{c|}{$\begin{array}{c}\text { IUGR Pregnancies } \\
\text { Group }(\mathbf{n = 1 0 0 )}\end{array}$} & P Value \\
& Mean & \pm SD & Mean & \pm SD & \\
Fetal Weight (gm) & 2622.00 & 189.94 & 1776.00 & 325.40 & $<0.001$ \\
$\begin{array}{c}\text { Placental Weight } \\
\text { (gm) }\end{array}$ & 521.30 & 58.36 & 366.07 & 46.46 & $<0.001$ \\
$\begin{array}{c}\text { Umbilical cord } \\
\text { length (cm) }\end{array}$ & 44.16 & 6.93 & 46.04 & 8.43 & $>0.05$ \\
\hline \multicolumn{7}{r}{ Table 3.1. Statistical Analysis of Significance } \\
\hline
\end{tabular}

\begin{tabular}{|cccc|}
\hline Variable & $\begin{array}{c}\text { Normal Pregnancies } \\
\text { Group }(\mathbf{n = 1 0 0})\end{array}$ & $\begin{array}{c}\text { IUGR Pregnancies } \\
\text { Group }(\mathbf{n = 1 0 0})\end{array}$ & P Value \\
Cord insertion & 38 & 29 & \\
Central & 25 & 22 & 0.05 \\
lateral & 18 & 35 & \\
Marginal & 19 & 14 & \\
Medial & Table 3.2. Statistical Analysis of Significance \\
\hline \multicolumn{3}{c}{} \\
\hline
\end{tabular}

\section{DISCUSSION}

In our study, the marginal attachment of umbilical cord with 0.25 insertion percentage was seen in 35 cases. The association between marginal attachment of the cord and low birth weight is statistically significant. The low birth weight may be explained by an altered distribution of foetal blood in the placenta as a result of different modes of arrangement of intra cotyledonary vessels of placentas of complicated pregnancy (Rath et al 1994) ${ }^{6}$. This vascular arrangement may be hampering equal distribution of blood flow in the placenta, increasing the risk to the mother and the foetus. Sonographic study could also clearly show the site of insertion of the umbilical cord of the placenta and in hypertensive mothers it was mostly marginal. This confirms the observations of Pretorius et al (1996) ${ }^{9}$ and Di Salvo et al (1998). ${ }^{10}$ In case the ultrasound report reveals marginal attachment, it is advisable to get it confirmed by colour Doppler imaging, if possible. According to S Biswas $2013^{11}$ velamentous insertion and marginal insertion of cord was a significant finding in the placentas of IUGR foetuses. Bjoro $\mathrm{K}$ Jr.,12 Biswas and Ghosh ${ }^{13}$ found that in the majority of IUGR cases, positions of insertions of umbilical cords were eccentric whereas in the control group, the majority was central.

\section{Umbilical Cord Length}

In the present study, difference in mean cord length in control and research group is insignificant. The $p$ value $(>0.05)$ is not significant. The study is consistent with study of S Kotgirwar et $\mathrm{al}^{3}$. Among western researchers, Figen Barut et $\mathrm{al}^{4}$ observed 
significant value $(\mathrm{p}<0.01)$ of mean cord length among research group. A length of at least $32 \mathrm{~cm}$ has been claimed necessary to prevent traction on the cord during vaginal delivery, so it is not surprising that shorter cords occasionally delay completion of the second stage of labour or cause placental abruptions, inversion of the uterus, cord herniation and cord rupture. Abnormally long cord increases the frequency of cord prolapsed, true knots, and coiling of the cord around foetal parts; all of which can produce foetal distress through cord compression. The mean length of umbilical cord varied in the present study between 30-60 cms in normal and abnormal pregnancies. No significant difference was seen in cord length according to type of pregnancy-normal or abnormal. From the literature review it was anticipated that there would be a wide range of umbilical cord lengths.

\section{Insertion of Umbilical Cord}

Perceival (1980) $)^{5}$ has reported that in $73 \%$ of cases, the site of insertion of umbilical cord is eccentric in position (somewhere between the centre and the edge of the placenta). Rath $G$ et al $(1994)^{6}$ reported that the marginal attachment of umbilical cord with 0-25 insertion percentage was seen in $27 \%$, which is higher by $7 \%$ as noted by Perceival (1980). ${ }^{5}$ The distribution of four categories of insertion of umbilical cord is almost equal in normotensive group. Even amongst the mild and moderate hypertensive subgroups, the distribution of four categories is almost similar. Only in the severe hypertensive cases the marginal attachment of umbilical cord with 0-25 insertion percentage score was the highest with $42 \%$. This high percentage in severe hypertension may be responsible for the low birth weight babies. Woods and Malan (1978) ${ }^{7}$ have studied 940 placentas and found no correlation between the birth weight and the site of cord insertion in normal term infants, however even in normotensive mothers the marginal attachment of umbilical cord was correlated with low birth weight babies. Besides the normotensive mothers, even in cases of mild, moderate and severe hypertensive cases, the marginal attachment of umbilical cord was related to low birth weight, most commonly noticed in the severe hypertensive subgroup. Shanklin $(1970)^{8}$ after studying 5000 placentas, observed a high degree of correlation between anomalous cord insertion and low birth weight.

\section{CONCLUSIONS}

It is noticed that the marginal insertion is associated with IUGR and that it can be diagnosed during antenatal check up by available techniques to further strengthen the proposed precautions to be taken during and after labour.

Financial or Other Competing Interests: None.

\section{REFERENCES}

[1] Pansky B. Review of medical embryology. New York: Macmillan 1982:72-5.

[2] Wulf KH. The placental insufficiency syndrome (a clinical concept) (author's transl). Z Geburtshilfe Perinatol 1981;185(1):2-11.

[3] Kotgirwar S, Ambiye M, Athavale S, et al. Study of gross and histological features of placenta in intrauterine growth retardation. J Anat Soc India 2011;60(1):37-40.

[4] Barut F, Barut A, Gun BD, et al. Intrauterine growth restriction and placental angiogenesis. Diagn Pathol 2010;5:24.

[5] Perceival R. Chorion and placenta with placental ischaemia. In: Holland and brews, manual of obstetrics. $14^{\text {th }}$ edn. Churchill Livingstone 1980:30-50.

[6] Rath G, Garg K, Anand C, et al. Vascular pattern of human placenta in complicated pregnancy, a corrosive cast study. Ann Nat Acad Med Sci 1994;30:17-22.

[7] Woods DL, Malan AF. The site of umbilical cord insertion and birth weight. BJOG: An International Journal of Obstetrics \& Gynaecology 1978;85(5):332-3.

[8] Shanklin DR. The influence of placental lesions on the newborn infant. Pediatr Clin North Am 1970;17(1):25-42.

[9] Pretorius DH, Chau C, Poeltler DM, et al. Placental cord insertion, visualization with prenatal ultrasonography. J Ultrasound Med 1996;15(8):585-93.

[10] Di Salvo DN, Benson CB, Laing FC, et al. Sonographic evaluation of the placental cord insertion site. Am J Roentgenol 1998;170(5):1295-8.

[11] Biswas S. Placental changes in idiopathic intrauterine growth restriction. OA Anatomy 2013;1(2):11.

[12] Bjoro K. Gross pathology of placenta in intrauterine growth retardation. Ann Chir Gynaecol 1981;70(6):31622.

[13] Biswas S, Ghosh SK. Gross morphological changes of placentas associated with intrauterine growth restriction of foetuses: a case control study. Early Hum Dev 2008;84(6):357-62. 